Editorial

\title{
100th Paper Milestone
}

\section{Gilbert Fantozzi}

INSA-Lyon, MATEIS Laboratory UMR CNRS 5510, 69621 Villeurbanne, France; gilbert.fantozzi@insa-lyon.fr

Received: 11 August 2020; Accepted: 30 August 2020; Published: 1 September 2020

The Ceramics journal has reached its first milestone with the publication of the 100th paper since its creation in 2018.

The 100 papers published by Ceramics concern the following topics:

- $\quad$ Novel Processing Routes of Ceramics for Functional Applications [1-6]

- Advances in the Field of Nanostructured Ceramic Composites [7-11]

- Functional Ceramics for Energy Applications [12-18]

- $\quad$ Ice Templated and Freeze Cast Ceramics [19-25]

- Ceramics for Biomedical Applications [26-34]

- $\quad$ Damage and Lifetime of Ceramic Matrix Composites [35-41]

- $\quad$ Advances in Luminescent Materials [42]

- $\quad$ Design, Properties, Damage and Lifetime of Refractory Ceramics [43-53]

- Advances in Structural Ceramic Materials [54-60]

- $\quad$ The Past, Present and Future of Additive Manufacturing [61]

- $\quad$ Design, Fabrication and Assessment of Ceramics for Advanced Optical Applications [62-67]

The paper that has received the highest number of citations is "Electrical Behavior and Microstructural Features of Electric Field-Assisted and Conventionally Sintered 3 mol.\% Yttria-Stabilized Zirconia" by Sabrina G.M. Carvalho, Eliana N.S. Muccillo and Reginaldo Muccillo.

Furthermore, the Ceramics journal has published eight Special Issues:

- Advances in the Field of Nanostructured Ceramic Composites edited by Laura Montanaro and Paola Palmero https://www.mdpi.com/journal/ceramics/special_issues/Advances_Nanostructured_ Ceramic_Composites

- Functional Ceramics for Energy Applications edited by Kyle G. Webber, Tor Grande and Mari-Ann Einarsrud https://www.mdpi.com/journal/ceramics/special_issues/functional_ceramics_ energy_applications

- Design, Fabrication and Assessment of Nanostructured Materials and Systems for Advanced Optical Applications edited by Alessandro Chiasera, Anna Lukowiak, Francesco Scotognella and Maurizio Ferrari https://www.mdpi.com/journal/ceramics/special_issues/nanostructured_ materials_optical_applications

- Novel Processing Routes of Ceramics for Functional Applications edited by Stephane Hocquet and Laurent Boilet https://www.mdpi.com/journal/ceramics/special_issues/processing_routes_ ceramics_functional_applications

- Ice-Templated and Freeze-Cast Ceramics edited by Sylvain Deville https://www.mdpi.com/journal/ ceramics/special_issues/ice_freeze

- Damage and Lifetime of Ceramic Matrix Composites edited by Gilbert Fantozzi, Nathalie Godin and Pascal Reynaud https://www.mdpi.com/journal/ceramics/special_issues/Damage_Lifetime_CMC

- Ceramics for Biomedical Applications edited by Laurent Gremillard and Jérôme Chevalier https://www.mdpi.com/journal/ceramics/special_issues/ceramics_biomedical 
- Design, Properties, Damage and Lifetime of Refractory Ceramics edited by Jacques Poirier https://www.mdpi.com/journal/ceramics/special_issues/refractory_ceramics

We should like to acknowledge all the authors of the published papers for their contribution. We want also to acknowledge the editors of the Special Issues for their significant work.

Now, we must continue to develop the Ceramics journal and encourage the submission of papers and the participation of board members. We want also to encourage young researchers to publish in the journal by creating a Ceramic Travel Award in 2020.

For this development, we need your confidence and your participation. I am counting on you to make a success of our Ceramics journal.

\section{References}

1. Renoirt, M.-S.; Maury, N.; Dupla, F.; Gonon, M. Structure and Properties of Piezoelectric Strontium Fresnoite Glass-Ceramics Belonging to the Sr-Ti-Si-Al-K-O System. Ceramics 2019, 2, 86-97. [CrossRef]

2. Muccillo, R.; Ferlauto, A.S.; Muccillo, E.N.S. Flash Sintering Samaria-Doped Ceria-Carbon Nanotube Composites. Ceramics 2019, 2, 64-73. [CrossRef]

3. Kobayashi, M.; Kato, H.; Miyazaki, T.; Kakihana, M. Hydrothermal Synthesis of Pseudocubic Rutile-Type Titania Particles. Ceramics 2019, 2, 56-63. [CrossRef]

4. Muccillo, R.; De Florio, D.Z.; Muccillo, E.N.S. Equimolar Yttria-Stabilized Zirconia and Samaria-Doped Ceria Solid Solutions. Ceramics 2018, 1, 343-352. [CrossRef]

5. Ahlhelm, M.; Werner, D.; Kaube, N.; Maier, J.; Abel, J.; Behnisch, T.; Moritz, T.; Michaelis, A.; Gude, M. Deriving Principles of the Freeze-Foaming Process by Nondestructive CT Macrostructure Analyses on Hydroxyapatite Foams. Ceramics 2018, 1, 65-82. [CrossRef]

6. Carvalho, S.G.; Muccillo, E.N.; Muccillo, R. Electrical Behavior and Microstructural Features of Electric Field-Assisted and Conventionally Sintered 3 mol\% Yttria-Stabilized Zirconia. Ceramics 2018, 1, 3-12. [CrossRef]

7. Montanaro, L.; Palmero, P. Advances in the Field of Nanostructured Ceramic Composites. Ceramics 2019, 2, 296-297. [CrossRef]

8. Gallardo-López, Á.; López-Pernía, C.; Muñoz-Ferreiro, C.; González-Orellana, C.; Morales-Rodríguez, A.; Poyato, R. Spark Plasma Sintered Zirconia Ceramic Composites with Graphene-Based Nanostructures. Ceramics 2018, 1, 153-164. [CrossRef]

9. Jiménez, M.; Samie, A.; Gadow, R.; Kern, F.; Bill, J. Siloxane Precursor-Based Protective Coatings for High Modulus Carbon Fibers in Ceramic Matrix Composites. Ceramics 2018, 1, 128-138. [CrossRef]

10. Gommeringer, A.; Kern, F.; Gadow, R. Enhanced Mechanical Properties in ED-Machinable Zirconia-Tungsten Carbide Composites with Yttria-Neodymia Co-Stabilized Zirconia Matrix. Ceramics 2018, 1, 26-37. [CrossRef]

11. Marchisio, A.; Tulliani, J.-M. Semiconducting Metal Oxides Nanocomposites for Enhanced Detection of Explosive Vapors. Ceramics 2018, 1, 98-119. [CrossRef]

12. Radan, K.; Kmet, B.; Drnovšek, S.; Prah, U.; Rojac, T.; Malič, B. Mechanochemically-Assisted Synthesis of Lead-Free Piezoelectric $\mathrm{CaZrO}_{3}$-Modified $(\mathrm{K}, \mathrm{Na}, \mathrm{Li})(\mathrm{Nb}, \mathrm{Ta}) \mathrm{O}_{3}$-Solid Solution. Ceramics 2018, 1, 304-318. [CrossRef]

13. Kimura, Y.; Kushi, T.; Unemoto, A.; Amezawa, K.; Kawada, T. Influence of Aging on Mechanical Properties of Yttria-Doped Zirconia. Ceramics 2018, 1, 287-303. [CrossRef]

14. Isogai, M.; Veber, A.; Cicconi, M.R.; Hayakawa, T.; De Ligny, D. Devitrification Behavior of Sol-Gel Derived $\mathrm{ZrO}_{2}-\mathrm{SiO}_{2}$ Rare-Earth Doped Glasses: Correlation between Structural and Optical Properties. Ceramics 2018, 1, 274-286. [CrossRef]

15. Harvey, S.P.; Ricote, S.; Diercks, D.R.; Jiang, C.-S.; Patki, N.S.; Manerbino, A.; Gorman, B.; Al-Jassim, M. Evolution of Copper Electrodes Fabricated by Electroless Plating on $\mathrm{BaZr}_{0.7} \mathrm{Ce}_{0.2} \mathrm{Y}_{0.1} \mathrm{O}_{3-\delta}$ Proton-Conducting Ceramic Membrane: From Deposition to Testing in Methane. Ceramics 2018, 1, 261-273. [CrossRef]

16. Xu, Y.; Zielke, P.; Van Nong, N.; Pirou, S.; Reolon, R.; Si, X.; Simonsen, S.B.; Norby, P.; Lühmann, H.; Bensch, W.; et al. Hydrothermal Synthesis, Characterization, and Sintering Behavior of Core-Shell Particles: A Principle Study on Lanthanum Strontium Cobaltite Coated with Nanosized Gadolinium Doped Ceria. Ceramics 2018, 1, 246-260. [CrossRef] 
17. Haugen, A.B.; Aguilera, L.M.; Kwok, K.; Molla, T.; Andersen, K.B.; Pirou, S.; Kaiser, A.; Hendriksen, P.V.; Kiebach, R. Exploring the Processing of Tubular Chromite- and Zirconia-Based Oxygen Transport Membranes. Ceramics 2018, 1, 229-245. [CrossRef]

18. Cordero, F. Elastic and Dielectric Evaluation of the Piezoelectric Response of Ferroelectrics Using Unpoled Ceramics. Ceramics 2018, 1, 211-228. [CrossRef]

19. Deville, S. Advances in Ice-Templated and Freeze-Casted Ceramics. Ceramics 2019, 2, 551-553. [CrossRef]

20. Gaudillere, C.; Garcia-Fayos, J.; Plaza, J.; Serra, J.M. Ice-Templating for the Elaboration of Oxygen Permeation Asymmetric Tubular Membrane with Radial Oriented Porosity. Ceramics 2019, 2, 246-259. [CrossRef]

21. Su, F.Y.; Mok, J.R.; McKittrick, J. Radial-Concentric Freeze Casting Inspired by Porcupine Fish Spines. Ceramics 2019, 2, 161-179. [CrossRef]

22. Papa, E.; Medri, V.; Natali Murri, A.; Miccio, F.; Landi, E. Ice-Templated Geopolymer-Fe/Mn Oxide Composites Conceived as Oxygen Carriers. Ceramics 2019, 2, 148-160. [CrossRef]

23. Ellis, S.N.; Romao, C.P.; White, M.A. Near-Zero Thermal Expansion in Freeze-Cast Composite Materials. Ceramics 2019, 2, 112-125. [CrossRef]

24. Rogers, C.; Pun, D.; Fu, Q.; Zhang, H. Fabricating MOF/Polymer Composites via Freeze Casting for Water Remediation. Ceramics 2018, 1, 353-363. [CrossRef]

25. Niksiar, P.; Su, F.Y.; Frank, M.B.; Ogden, T.A.; Naleway, S.E.; Meyers, M.A.; McKittrick, J.; Porter, M.M. External Field Assisted Freeze Casting. Ceramics 2019, 2, 208-234. [CrossRef]

26. Frigan, K.; Chevalier, J.; Zhang, F.; Spies, B.C. Is a Zirconia Dental Implant Safe When It Is Available on the Market? Ceramics 2019, 2, 568-577. [CrossRef]

27. Pezzotti, G.; Marin, E.; Zanocco, M.; Boschetto, F.; Zhu, W.; McEntire, B.J.; Bal, B.S.; Adachi, T.; Yamamoto, T.; Kanamura, N.; et al. Osteogenic Enhancement of Zirconia-Toughened Alumina with Silicon Nitride and Bioglass ${ }^{\circledR}$. Ceramics 2019, 2, 554-567. [CrossRef]

28. Ali, M.; Al-Hajjar, M.; Fisher, J.; Jennings, L.M. The Influence of Kinematic Conditions and Variations in Component Positioning on the Severity of Edge Loading and Wear of Ceramic-on-Ceramic Hip Bearings. Ceramics 2019, 2, 488-501. [CrossRef]

29. Siniscalco, D.; Dutreilh-Colas, M.; Hjezi, Z.; Cornette, J.; El Felss, N.; Champion, E.; Damia, C. Functionalization of Hydroxyapatite Ceramics: Raman Mapping Investigation of Silanization. Ceramics 2019, 2, 372-384. [CrossRef]

30. Kern, F. Evidence of Phase Transitions and Their Role in the Transient Behavior of Mechanical Properties and Low Temperature Degradation of 3Y-TZP Made from Stabilizer-Coated Powder. Ceramics 2019, 2, 271-285. [CrossRef]

31. Engelmann, T.; Desante, G.; Labude, N.; Rütten, S.; Telle, R.; Neuss, S.; Schickle, K. Coatings Based on Organic/Non-Organic Composites on Bioinert Ceramics by Using Biomimetic Co-Precipitation. Ceramics 2019, 2, 260-270. [CrossRef]

32. Suchanek, K.; Perzanowski, M.; Lekki, J.; Strą, M.; Marszałek, M. Ammonium Hydroxide Mediated Hydrothermal Crystallization of Hydroxyapatite Coatings on Titanium Substrate. Ceramics 2019, 2, 180-189. [CrossRef]

33. Safronova, T.; Putlayev, V.; Filippov, Y.; Shatalova, T.; Karpushkin, E.; Larionov, D.; Kazakova, G.; Shakhtarin, Y. Calcium Phosphate Powder Synthesized from Calcium Acetate and Ammonium Hydrophosphate for Bioceramics Application. Ceramics 2018, 1, 375-392. [CrossRef]

34. Fabris, D.; Lasagni, A.F.; Fredel, M.C.; Henriques, B. Direct Laser Interference Patterning of Bioceramics: A Short Review. Ceramics 2019, 2, 578-586. [CrossRef]

35. Lamon, J. Static Fatigue of SiC Multifilament Tows at Temperatures up to $120{ }^{\circ} \mathrm{C}$ in Air. Ceramics 2019, 2, 426-440. [CrossRef]

36. Panakarajupally, R.P.; Presby, M.J.; Manigandan, K.; Zhou, J.; Chase, G.G.; Morscher, G.N. Thermomechanical Characterization of SiC/SiC Ceramic Matrix Composites in a Combustion Facility. Ceramics 2019, 2, 407-425. [CrossRef]

37. Dassios, K.G.; Matikas, T.E. Assessment of Fatigue Damage and Crack Propagation in Ceramic Matrix Composites by Infrared Thermography. Ceramics 2019, 2, 393-406. [CrossRef]

38. Bache, M.R.; Newton, C.D.; Jones, J.P.; Pattison, S.; Gale, L.; Nicholson, P.I.; Weston, E. Advances in Damage Monitoring Techniques for the Detection of Failure in $\mathrm{SiC}_{\mathrm{f}} / \mathrm{SiC}$ Ceramic Matrix Composites. Ceramics 2019, 2, 347-371. [CrossRef] 
39. Li, L. Effect of Cyclic Fatigue Loading on Matrix Multiple Fracture of Fiber-Reinforced Ceramic-Matrix Composites. Ceramics 2019, 2, 327-346. [CrossRef]

40. Perrot, G.; Couégnat, G.; Ricchiuto, M.; Vignoles, G.L. Image-Based Numerical Modeling of Self-Healing in a Ceramic-Matrix Minicomposite. Ceramics 2019, 2, 308-326. [CrossRef]

41. Gadow, R.; Weichand, P.; Jiménez, M. Process Technology, Applications and Thermal Resistivity of Basalt Fiber Reinforced SiOC Composites. Ceramics 2019, 2, 298-307. [CrossRef]

42. Einbergs, E.; Zolotarjovs, A.; Bite, I.; Laganovska, K.; Auzins, K.; Smits, K.; Trinkler, L. Usability of Cr-Doped Alumina in Dosimetry. Ceramics 2019, 2, 525-535. [CrossRef]

43. Loison, L.; Sassi, M.; Tonnesen, T.; De Bilbao, E.; Telle, R.; Poirier, J. Differences in the Corrosive Spalling Behavior of Alumina-Rich Castables: Microstructural and Crystallographic Considerations of Alumina and Calcium Aluminate Matrices. Ceramics 2020, 3, 223-234. [CrossRef]

44. Unterreiter, G.; Kreuzer, D.R.; Lorenzoni, B.; Marschall, H.U.; Wagner, C.; Machhammer, R.; Hackl, G. Compressive Creep Measurements of Fired Magnesia Bricks at Elevated Temperatures Including Creep Law Parameter Identification and Evaluation by Finite Element Analysis. Ceramics 2020, 3, 210-222. [CrossRef]

45. Ali, M.; Sayet, T.; Gasser, A.; Blond, E. Transient Thermo-Mechanical Analysis of Steel Ladle Refractory Linings Using Mechanical Homogenization Approach. Ceramics 2020, 3, 171-189. [CrossRef]

46. Wöhrmeyer, C.; Gao, J.; Parr, C.; Szepizdyn, M.; Mineau, R.-M.; Zhu, J. Corrosion Mechanism of A Density-Reduced Steel Ladle Lining Containing Porous Spinel-Calcium Aluminate Aggregates. Ceramics 2020, 3, 155-170. [CrossRef]

47. Pacheco, G.; Gonçalves, G.E.; Lins, V. Qualitative and Quantitative Coating Tests: A Comparison in Magnesia-Spinel Refractory Bricks. Ceramics 2020, 3, 144-154. [CrossRef]

48. De Villiers, J.P.; Mulange, D.; Garbers-Craig, A.M. The Effect of Titanium Oxide Additions on the Phase Chemistry and Properties of Chromite-Magnesia Refractories. Ceramics 2020, 3, 127-143. [CrossRef]

49. Reichert, W.; Nießen, J.; Leto, P.; Etzold, S.; Kröll, E.; Tonnesen, T.; Telle, R. Correlation of Thermo-Elastic Material and Corrosion Behavior of Refractory Castables by In-Situ Measurements. Ceramics 2020, 3, 101-113. [CrossRef]

50. Peng, H.; Liu, J.; Wang, Q.; Li, Y. Improvement in Slag Resistance of No-Cement Refractory Castables by Matrix Design. Ceramics 2020, 3, 31-39. [CrossRef]

51. Dudczig, S.; Schmidt, G.; Aneziris, C.G.; Wöhrmeyer, C.; Parr, C.; Gehre, P. Corrosion of MgO-C with Magnesium Aluminate Spinel Addition in A Steel Casting Simulator. Ceramics 2020, 3, 12-21. [CrossRef]

52. Hopp, V.; Masoudi Alavi, A.; Sax, A.; Quirmbach, P. Influence of Aluminium and Boron Orthophosphate on the Setting and the Resulting Structure of Alkali Silicate Binders for Refractory Application. Ceramics 2020, 3 , 1-11. [CrossRef]

53. Chotard, T.; Arbelaez Morales, L.; Bouchetou, M.-L.; Poirier, J. Thermomechanical Characterisation of Mullite Zirconia Composites Sintered from Andalusite for High Temperature Applications. Ceramics 2019, 2, 587-601. [CrossRef]

54. Günther, A.; Moritz, T.; Mühle, U. Microstructure and Interface Characteristics of 17-4PH/YSZ Components after Co-Sintering and Hydrothermal Corrosion. Ceramics 2020, 3, 245-257. [CrossRef]

55. Guo, R.; Wang, Q.; Bao, J.; Song, X. Preparation and Properties of Co-Doped Magnesium Lanthanum Hexaluminat Blue Ceramics. Ceramics 2020, 3, 235-244. [CrossRef]

56. Gommeringer, A.; Kern, F. Mechanical Properties and Electrical Discharge Machinability of Alumina-10 vol\% Zirconia-28 vol\% Titanium Nitride Composites. Ceramics 2020, 3, 199-209. [CrossRef]

57. Kern, F. Properties of 2 mol\% Yttria Stabilized Zirconia-Alumina-Cerium Hexaaluminate Composites. Ceramics 2020, 3, 190-198. [CrossRef]

58. Gallardo-López, Á.; Castillo-Seoane, J.; Muñoz-Ferreiro, C.; López-Pernía, C.; Morales-Rodríguez, A.; Poyato, R. Flexure Strength and Fracture Propagation in Zirconia Ceramic Composites with Exfoliated Graphene Nanoplatelets. Ceramics 2020, 3, 78-91. [CrossRef]

59. Díaz, M.; Smirnov, A.; Gutiérrez-González, C.; Estrada, D.; Bartolomé, J.F. Microstructure and Mechanical Properties of Zirconia (3Y-TZP)/Zr Composites Prepared by Wet Processing and Subsequent Spark Plasma Sintering. Ceramics 2020, 3, 53-64. [CrossRef]

60. Balazsi, K.; Furkó, M.; Klimczyk, P.; Balázsi, C. Influence of Graphene and Graphene Oxide on Properties of Spark Plasma Sintered $\mathrm{Si}_{3} \mathrm{~N}_{4}$ Ceramic Matrix. Ceramics 2020, 3, 40-50. [CrossRef] 
61. Chavez, L.A.; Ibave, P.; Wilburn, B.; Alexander, D., IV; Stewart, C.; Wicker, R.; Lin, Y. The Influence of Printing Parameters, Post-Processing, and Testing Conditions on the Properties of Binder Jetting Additive Manufactured Functional Ceramics. Ceramics 2020, 3, 65-77. [CrossRef]

62. Meroni, C.; Scotognella, F.; Boucher, Y.; Lukowiak, A.; Ristic, D.; Speranza, G.; Varas, S.; Zur, L.; Ivanda, M.; Taccheo, S.; et al. Low-Threshold Coherent Emission at $1.5 \mu \mathrm{m}$ from Fully $\mathrm{Er}^{3+}$ Doped Monolithic 1D Dielectric Microcavity Fabricated Using Radio Frequency Sputtering. Ceramics 2019, 2, 74-85. [CrossRef]

63. Gerardino, A.; Pettinari, G.; Caselli, N.; Vignolini, S.; Riboli, F.; Biccari, F.; Felici, M.; Polimeni, A.; Fiore, A.; Gurioli, M.; et al. Coupled Photonic Crystal Nanocavities as a Tool to Tailor and Control Photon Emission. Ceramics 2019, 2, 34-55. [CrossRef]

64. Głuchowski, P.; Oganisian, K.; Tomala, R.; Łukowiak, A.; Karpinsky, D.; Alikin, D.; Kholkin, A.; Stręk, W. Optical, Dielectric and Magnetic Properties of $\mathrm{La}_{1-x} \mathrm{Nd}_{x} \mathrm{FeO}_{3}$ Powders and Ceramics. Ceramics 2019, 2, 1-12. [CrossRef]

65. Vermillac, M.; Lupi, J.-F.; Trzesien, S.; Ude, M.; Blanc, W. On the Enlargement of the Emission Spectra from the ${ }^{4} \mathrm{I}_{13 / 2}$ Level of $\mathrm{Er}^{3+}$ in Silica-Based Optical Fibers through Lanthanum or Magnesium Co-Doping. Ceramics 2018, 1, 364-374. [CrossRef]

66. Kriegel, I.; Guizzardi, M.; Scotognella, F. Tantalum Arsenide-Based One-Dimensional Photonic Structures. Ceramics 2018, 1, 139-144. [CrossRef]

67. Laurikenas, A.; Beganskiene, A.; Kareiva, A. On the Synthesis and Characterization of Lanthanide Metal-Organic Frameworks. Ceramics 2018, 1, 54-64. [CrossRef]

(C) 2020 by the author. Licensee MDPI, Basel, Switzerland. This article is an open access article distributed under the terms and conditions of the Creative Commons Attribution (CC BY) license (http://creativecommons.org/licenses/by/4.0/). 